\title{
From Nominal to True A Posteriori Probabilities: An Exact Bayesian Theorem Based Probabilistic Data Association Approach for Iterative MIMO Detection and Decoding
}

\author{
Shaoshi Yang, Member, IEEE, Tiejun Lv, Senior Member, IEEE, Robert G. Maunder, Senior Member, IEEE, \\ and Lajos Hanzo, Fellow, IEEE
}

\begin{abstract}
It was conventionally regarded that the approximate Bayesian theorem based existing probabilistic data association (PDA) algorithms output the estimated symbol-wise $a$ posteriori probabilities (APPs) as soft information. In our recent work, however, we demonstrated that these probabilities are not the true APPs in the rigorous mathematical sense, but a type of nominal APPs, which are unsuitable for the classic architecture of iterative detection and decoding (IDD) aided receivers. To circumvent this predicament, in this paper we propose an exact Bayesian theorem based logarithmic domain PDA (EB-Log-PDA) method, whose output has similar characteristics to the true APPs, and hence it is readily applicable to the classic IDD architecture of multiple-input-multiple-output (MIMO) systems using the general $M$-ary modulation. Furthermore, we investigate the impact of the EB-Log-PDA algorithm's inner iteration on the design of EB-Log-PDA aided IDD receiver. We demonstrate that introducing inner iterations into EB-Log-PDA, which is common practice in conventional-PDA aided uncoded MIMO systems, would actually degrade the IDD receiver's performance, despite significantly increasing the overall computational complexity of the IDD receiver. Finally, we investigate the relationship between the extrinsic log-likelihood ratios (LLRs) of the proposed EB-Log-PDA and of the approximate Bayesian theorem based logarithmic domain PDA (AB-Log-PDA) reported in our previous work. Despite their difference in extrinsic LLRs, we also show that the IDD schemes employing the EB-Log-PDA and the ABLog-PDA without incorporating any inner PDA iterations have a similar achievable performance close to that of the optimal maximum a posteriori (MAP) detector based IDD receiver, while imposing a significantly lower computational complexity in the scenarios considered.
\end{abstract}

The associate editor coordinating the review of this paper and approving it for publication was Prof. E. Ayanoglu. Manuscript received June 21, 2012; revised February 12, 2013

The financial support of the China Scholarship Council (CSC), of the Research Councils UK (RCUK) under the India-UK Advanced Technology Center (IU-ATC), of the EU under the auspices of the Concerto project, of the EPSRC under the grant EP/J015520/1, of the National Natural Science Foundation of China (NSFC) (Grant No. 61271188) and of the European Research Councils Advanced Fellow Grant is gratefully acknowledged.

S. Yang is with the School of Electronics and Computer Science, University of Southampton, Southampton, SO17 1BJ, UK, and also with the School of Information and Communication Engineering, Beijing University of Posts and Telecommunications, Beijing, 100876, China (e-mail: shaoshi_yang@ieee.org).

$\mathrm{T}$. Lv is with the School of Information and Communication Engineering, Beijing University of Posts and Telecommunications, Beijing, 100876, China (e-mail: 1vtiejun@bupt.edu.cn).

R. G. Maunder and L. Hanzo are with the School of Electronics and Computer Science, University of Southampton, Southampton, SO17 1BJ, UK (e-mail: $\{$ rm, lh\}@ecs.soton.ac.uk).

Digital Object Identifier 10.1109/TCOMM.2013.09.120427
Index Terms-Bayesian Theorem, iterative detection and decoding, multiple-input-multiple-output (MIMO), probabilistic data association (PDA), $M$-ary modulation.

\section{INTRODUCTION}

$\mathbf{T}$ HE probabilistic data association (PDA) filter technique constitutes a successful statistical approach to the plot-to-track association problem of radar systems [1], [2] and to the visual target tracking problem in the field of computer vision [3]. Recently, it has also been invoked for low-complexity, high-performance soft-inputsoft-output (SISO) detection/equalization in multiple-inputmultiple-output (MIMO) fading channels [4]-[10]. In this scenario, the probabilities of the potential candidate symbols serve as the soft input/output information and are typically estimated using an approximate version of the Bayesian formula relying on a self-iterative process. The key operation in this process is the iterative approximation of the interferenceplus-noise term obeying a Gaussian mixture distribution as an ever-updated multivariate Gaussian distribution [4]-[10].

Other alternative SISO MIMO detection algorithms include (but are not limited to) the high-complexity maximum $a$ posteriori (MAP) algorithm, the family of soft interference cancellation algorithms [11], [12], and the list sphere decoding (LSD) algorithm [13]. More explicitly, the MAP algorithm achieves the best performance, but its computational complexity increases exponentially with the number of transmit antennas. Compared with the MAP algorithm, the LSD has a suboptimal performance with reduced complexity. However, the size of the LSD's candidate list is determined by the SNR-dependent sphere radius, which still results in an excessive complexity for the scenario of low-SNR and/or highthroughput MIMO systems [14], especially in the vicinity of the "turbo-cliff". The soft interference cancellation algorithm has a substantially lower computational complexity than the MAP and the LSD algorithms, but its achievable performance is also less attractive. Since the PDA algorithm was shown to achieve a near-optimal performance at a low computational complexity in uncoded systems [4], it is interesting to investigate its potential in coded MIMO systems.

The output symbol probabilities of the conventional PDA detectors/equalizers were widely regarded as a posteriori 
probabilities (APPs) without causing any notable problems [4]-[10]. This is because i) they qualify as some form of a posteriori estimates based on the a priori known received signal and the channel matrix, and hence are typically treated as the true APPs; ii) they are eminently applicable to numerous applications, including the multiuser detection (MUD) of uncoded code-division multiple-access (CDMA) systems [4], the multistream symbol detection of uncoded MIMO systems [5], [9], the soft decision equalization of frequency-selective MIMO channels [6]-[8], as well as the distributed soft reception in base station cooperation [10]; iii) additionally, the equivalent bit-wise log-likelihood ratios (LLRs) generated from these probabilities are also applicable to forward error correction (FEC)-coded MIMO systems employing the traditional open loop receiver architecture, in which the PDA detector is not required to generate bit-wise extrinsic LLRs [15].

However, as pointed out in [16], [17] that upon considering the more powerful iterative detection and decoding (IDD) receiver architecture of high-throughput $M$-ary modulation aided MIMO systems, the IDD receiver design using the PDA based algorithms becomes challenging if the estimated output symbol-wise probabilities are still treated as the true APPs.

First of all, it was not clear how to produce the correct extrinsic LLRs required by the outer FEC decoder with respect to the approximate Bayesian theorem based existing PDA detectors until very recently [16], [17]. Although an IDD scheme employing the existing PDA detector was proposed in [18], it was essentially an MAP detector based IDD arrangement using a hard PDA detector for generating the candidate-search list, and the PDA did not generate the true APPs for channeldecoding. In line with the conventional interpretation, namely that the symbol-wise output probabilities of the existing PDA detectors are APPs, one may assume that a plausible technique of generating the bit-wise extrinsic LLRs at the output of PDA detectors is to subtract the bit-wise a priori LLRs from the bitwise $a$ posteriori LLRs generated from the estimated symbolwise probabilities of the existing PDAs. Unfortunately, this classic relationship no longer holds for the existing PDAs, because it was shown in [16], [17] and will be demonstrated further in Section III that the symbol-wise probabilities which act as the output of the existing PDAs - are actually not the true APPs, but some sort of nominal APPs. Surprisingly, these nominal APPs had been regarded as the true APPs in the open literature [4]-[10] for a long time, and the distinctions between these nominal APPs and the true APPs were never noticed until recently [16], [17], where it was shown that this dilemma may be potentially misleading for designing IDD receivers relying on the existing PDA based methods.

Secondly, the family of PDA based detectors have a selfiterative structure in uncoded systems, where the estimated symbol-wise nominal APPs are delivered to the next inner iteration after the current inner iteration is completed. It has been revealed that introducing inner iterations into our previously proposed approximate Bayesian theorem based logarithmic domain PDA (AB-Log-PDA) [16], [17] actually degrades the achievable performance of the IDD receiver, which is in contrast to the beneficial impact of PDA detector's inner iterations in uncoded systems. However, when an IDD scheme based on other type of PDA algorithm, if any, is considered, the question of how to configure the inner iterations of this type of PDA remains an open problem.

Against this background, in this paper we aim for the indepth characterization of the existing PDAs' output and then for designing a novel PDA approach, whose output exhibits the main properties of the true APPs. As a benefit, the proposed PDA approach becomes immediately applicable to the classic IDD architecture. The main contributions of this paper are as follows.

1) We propose an exact Bayesian theorem based logarithmic domain PDA (EB-Log-PDA) approach for the IDD scheme of FEC-coded MIMO systems using arbitrary $M$-ary modulation for transmission over fading MIMO channels. As opposed to that of the existing PDAs based on an approximate version of the Bayesian theorem [4]-[10], [16], [17], the estimated symbol-wise output probabilities of the EB-Log-PDA exhibit similar characteristics to the true APPs. Hence the bit-wise extrinsic LLRs delivered by the proposed EB-Log-PDA to the outer FEC decoder may be calculated simply by subtracting the bit-wise a priori LLRs from the bit-wise a posteriori LLRs, which are generated from the symbol-wise output APPs of the EB-Log-PDA. Furthermore, in contrast to the existing probabilistic-domain PDAs [4]-[10], the proposed EB-LogPDA based detector operates in the logarithmic domain, which results in a better numerical stability and a higher numerical accuracy in the context of IDD receivers.

2) We investigate the relationship between the EB-LogPDA based IDD scheme and our previously proposed ABLog-PDA aided IDD scheme [16], [17]. We demonstrate that the two schemes achieve a similar performance when dispensing with inner iterations within the PDAs. However, this does not mean that the extrinsic LLRs output by the two PDAs are identical. Actually, the EB-Log-PDA may be viewed as a scheme using the same a priori information twice in different processing blocks, whereas the AB-LogPDA utilizes the corresponding a priori information once. Our analysis of the cumulative distribution functions (CDFs) and the probability density functions (PDFs) concerning the differences between the two types of extrinsic LLRs reveal that these two types of extrinsic LLRs are different, even though they might be similar in certain scenarios.

3) We investigate the impact of the inner iterations within the EB-Log-PDA on the achievable performance of the corresponding IDD scheme. We will demonstrate that, similar to the scenario of the AB-Log-PDA, the performance of the EB-Log-PDA-based IDD scheme is also degraded when inner iterations of the EB-Log-PDA are invoked. More specifically, the performance of the EB-Log-PDA based IDD scheme is consistently degraded as the number of inner iterations within the EB-Log-PDA is increased. By contrast, the performance degradation profile of the AB-Log-PDA based IDD scheme exhibits fluctuations upon increasing the number of inner iterations within the AB-Log-PDA. The reasons accounting for these phenomena are discussed as well.

4) When dispensing with inner iterations within both the EB-Log-PDA and the AB-Log-PDA in the context of our IDD architecture, the resultant IDD receiver exhibits a performance close to that of the optimal MAP-based IDD scheme, while imposing a significantly lower computational complexity in 
the scenarios considered.

The remainder of the paper is organized as follows. In Section II, our FEC-coded MIMO system model is detailed. In Section III, the proposed EB-Log-PDA relying on an external source of a priori soft information is presented. Then, in Section IV, the method of calculating the extrinsic LLRs for the EB-Log-PDA is derived, and the relationship between the extrinsic LLRs of the EB-Log-PDA and that of the ABLog-PDA is elucidated as well. Our simulation results and discussions are provided in Section V, while our conclusions are offered in Section VI.

\section{SySTEM Model}

Similar to [16], [17], we consider the FEC-coded spatial multiplexing MIMO system of Fig. 1. At the transmitter end, the $\left(L_{f} \times 1\right)$-bit source frame $\mathbf{x}_{1}$ is first encoded by a rate $R<1$ FEC encoder (typically a convolutional code, a turbo code or an LDPC code) into the $\left(\frac{L_{f}}{R} \times 1\right)$-bit coded frame $\mathbf{c}_{1}$. In order to guard against bursty fading, $\mathbf{c}_{1}$ is then passed through a bit-interleaver. Then, the $\left(\frac{L_{f}}{R} \times 1\right)$-bit interleaver's output frame $\mathbf{d}_{2}$ is mapped into the $\left(\frac{L_{f}}{R M_{b}} \times 1\right)$-element symbol frame $\mathbf{s}_{2}$, with each symbol taken from a modulation constellation $\mathcal{A}=\left\{a_{1}, a_{2}, \cdots, a_{M}\right\}$, where $M_{b}=\log _{2} M$ is the number of bits per symbol. Finally, $\mathbf{s}_{2}$ is transmitted in the form of the $\left(N_{t} \times 1\right)$-element symbol vector $\mathbf{s}$ using $N_{t} \geq 1$ transmit antennas per channel use, ${ }^{1}$ and $\mathbf{s}$ does not contain any additional space-time coding. At the output of the fading channel $\mathbf{H}$, the received $\left(N_{r} \times 1\right)$-element complexvalued baseband signal vector per channel use is represented by

$$
\mathbf{y}=\mathbf{H s}+\mathbf{n},
$$

where $\mathbf{s}=\left[s_{1}, s_{2}, \cdots, s_{N_{t}}\right]^{T}$ is normalized by the componentwise energy constraint $\mathbb{E}\left(\left|s_{i}\right|^{2}\right)=E_{\mathbf{s}} / N_{t}$ in order to ensure a total transmit energy of $E_{\mathbf{s}}$ per channel use; $\mathbf{n}$ is the $\left(N_{r} \times 1\right)$-element zero-mean complex-valued circularly symmetric Gaussian noise vector having a covariance matrix of $2 \sigma^{2} \mathbf{I}_{N_{r}}$, where $\mathbf{I}_{N_{r}}$ represents an $\left(N_{r} \times N_{r}\right)$-element identity matrix; and $\mathbf{H}$ is an $\left(N_{r} \times N_{t}\right)$-element complex-valued matrix with entries of $h_{j i}$, which are perfectly known to the receiver, $j=1, \cdots, N_{r}, i=1, \cdots, N_{t}, N_{r} \geq N_{t}$. In this paper, we assume that

$$
h_{j i}=r \exp (j \theta)
$$

is independent and identically distributed (i.i.d), where the phase $\theta$ is uniformly distributed and independent of the envelope $r$, while $r$ obeys the Nakagami- $m$ distribution with the probability density function (PDF) of [19]

$$
p(r)=\frac{2}{\Gamma(m)}\left(\frac{m}{\Omega}\right)^{m} r^{2 m-1} \exp \left(-m r^{2} / \Omega\right), r \geq 0
$$

where $\Gamma(\cdot)$ represents the Gamma function, $\Omega \triangleq \mathbb{E}\left(r^{2}\right)$, and the fading parameter is defined as $m \triangleq \Omega^{2} / \mathbb{E}\left[\left(r^{2}-\Omega\right)^{2}\right]$, $m \geq 0.5$. Note that the Nakagami- $m$ fading model captures a wide range of realistic fading environments, encompassing the most frequently used Rayleigh fading model as a special case and often serving as a good approximation to the Rician fading

\footnotetext{
${ }^{1}$ This indicates that a single coded bit frame $\mathbf{c}_{1}$ is transmitted by $\frac{L_{f}}{R M_{b} N_{t}}$ channel uses.
}

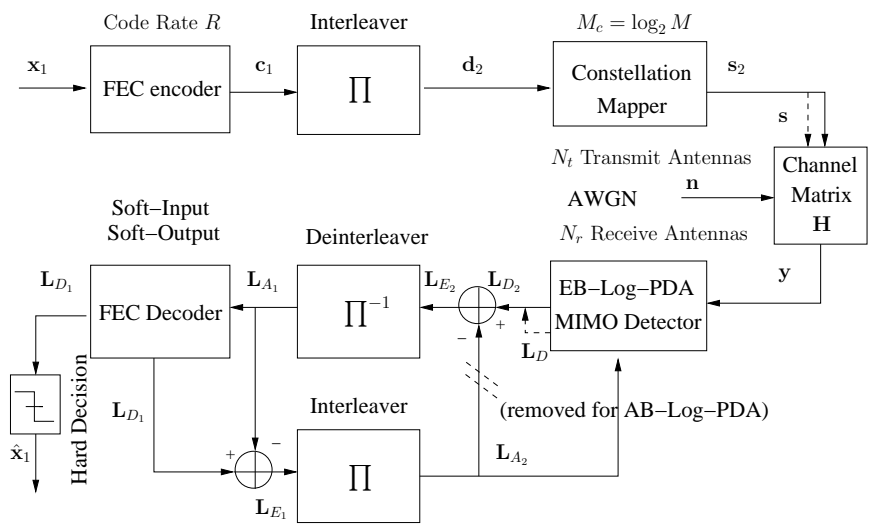

Fig. 1. FEC-coded MIMO system with PDA methods based iterative detection and decoding receiver. The subscript " 1 " denotes the processing modules associated with the "outer" FEC encoder/decoder, and the subscript "2" denotes the processing modules that are connected with the "inner" spacetime mapper/detector. The arrow with dashed line indicates that $\mathbf{s}$ and $\mathbf{L}_{D}$ are the subvectors of $\mathbf{s}_{2}$ and $\mathbf{L}_{D_{2}}$, respectively. The closed loop of $\mathbf{L}_{E_{2}} \rightarrow$ $\mathbf{L}_{A_{1}} \rightarrow \mathbf{L}_{E_{1}} \rightarrow \mathbf{L}_{A_{2}} \rightarrow \mathbf{L}_{E_{2}}$ represents an outer iteration compared to the inner iteration inside the soft FEC decoder or the soft MIMO detector.

model. ${ }^{2}$ More specifically, as $m$ becomes smaller, the fading effect becomes more severe. For example, when $m$ decreases to 0.5 , Eq. (3) approaches the one-sided Gaussian distribution; when $m=1$, Eq. (3) reduces to a Rayleigh PDF, and as $m \rightarrow \infty$, Eq. (3) reduces to a $\delta$-distribution located at $r=1$, which corresponds to no fading of the amplitude, but imposing a "pure random phase" obeying a uniform distribution on the circle of radius $\sqrt{\Omega}$.

\section{The EB-Log-PDA with A PRIORI Soft FeEdback FROM THE FEC DECODER}

In contrast to the AB-Log-PDA proposed in [16], [17], where the so-called non-decoupled signal model was used, below we use the decoupled signal model in order to further reduce the computational complexity. ${ }^{3}$ When relying on the zero-forcing principle based preprocessing, the received signal model of (1) may be rewritten as

$$
\tilde{\mathbf{y}}=\mathbf{s}+\tilde{\mathbf{n}}=s_{i} \mathbf{e}_{i}+\underbrace{\sum_{k \neq i} s_{k} \mathbf{e}_{k}}_{\mathbf{u}_{i}}+\tilde{\mathbf{n}} \triangleq s_{i} \mathbf{e}_{i}+\underbrace{\mathbf{u}_{i}+\tilde{\mathbf{n}}}_{\mathbf{v}_{i}},
$$

\footnotetext{
${ }^{2}$ The Rician and the Nakagami- $m$ models may be deemed to behave similarly near their mean value. Hence, for the sake of analytical simplicity, the Nakagami- $m$ model is often advocated in the literature as an approximation of the Rician model. This approximation is more accurate if the main lobe of the Rician model's PDF is concerned, but it becomes inaccurate for the tail of the Rician model's PDF. Since bit errors or outages mainly occur during deep fades, these performance measures are typically governed by the tail of the PDF, which represents the probability of receiving a low power. As a result, for deep fades, modeling a Rician fading signal by a Nakagami distribution of the amplitude leads to overly optimistic results.

${ }^{3}$ In the non-decoupled signal model, (1) is directly expanded as $\mathbf{y}=s_{i} \mathbf{h}_{i}+$ $\sum_{k \neq i} s_{k} \mathbf{h}_{k}+\mathbf{n}$, where $\mathbf{h}_{i}$ is the $i$ th column of $\mathbf{H}, i, k=1,2, \cdots, N_{t}$. As will be demonstrated in Section V-D, the computational complexity of the non-decoupled signal model based PDA method is related to the modulation order $M$, to the number of transmit antennas $N_{t}$ as well as to the number of receive antennas $N_{r}$. By contrast, the computational complexity of the decoupled signal model based PDA is related only to the modulation order $M$ and the number of the transmit antennas $N_{t}$. This is particularly important for spatial multiplexing MIMO systems, where $N_{r} \geq N_{t}$ is typically required.
} 
where we have $\tilde{\mathbf{y}}=\left(\mathbf{H}^{H} \mathbf{H}\right)^{-1} \mathbf{H}^{H} \mathbf{y}, \tilde{\mathbf{n}}=\left(\mathbf{H}^{H} \mathbf{H}\right)^{-1} \mathbf{H}^{H} \mathbf{n}$, $s_{i}$ is the $i$ th element of $\mathbf{s}$, and $\mathbf{e}_{i}$ denotes an $\left(N_{t} \times 1\right)$ element vector, whose $i$ th element is equal to one and the other elements are equal to zero, $i, k=1,2, \cdots, N_{t}$. Note that although the term $\mathbf{u}_{i}$ in (4) appears as the sum of $\left(N_{t}-1\right)$ inter-antenna interference (IAI) components, it is in fact orthogonal to the term $s_{i} \mathbf{e}_{i}$, which implies that $\mathbf{u}_{i}$ does not impose interference on $s_{i}$. However, it is the interference-plusnoise term $\mathbf{v}_{i}$ that contaminates the detection of the symbol $s_{i}$.

For uncoded MIMO systems, where no outer source of a priori soft information about the transmitted symbols is available, the existing PDA methods typically use the received signal $\mathbf{y}$ and the channel matrix $\mathbf{H}$ as input quantities, and then generate the estimated symbol-wise nominal APPs of the transmitted symbols $\left\{s_{i}\right\}_{i=1, \cdots, N_{t}}$ as its output. By contrast, for FEC-coded MIMO systems, we have an extra input quantity, which is the soft information feedback gleaned from the outer FEC decoder. In this scenario, because the reliability of the FEC decoder's output is typically higher than that of the soft MIMO detector at the previous stage, some of the key operations of the proposed EB-Log-PDA are implemented in the logarithmic domain in order to improve the achievable numerical stability and accuracy.

Although the interference-plus-noise term $\mathbf{v}_{i}$ obeys a multimodal Gaussian mixture distribution [15]-[17], initially it is plausible to obtain a coarse estimate of $s_{i}$ by assuming that $\mathbf{v}_{i}$ obeys a single $N_{t}$-variate Gaussian distribution. It is worth noting that in the circumstances considered each element of $\mathbf{v}_{i}$ is the sum of only two scalar random variables for any $N_{t} \geq 2$, hence, according to the central limit theorem, the Gaussian approximation of $\mathbf{v}_{i}$ does not become more accurate when $N_{t}$ is increased. ${ }^{4}$ This trend is different from that of the non-decoupled signal model based PDA [16], [17]. In order to fully characterize the complex random vector $\mathbf{v}_{i}$ which is not necessarily proper $^{5}$ [20], [21], we specify the mean of

$$
\boldsymbol{\mu}_{i} \triangleq \mathbb{E}\left(\mathbf{v}_{i}\right)=\sum_{k \neq i} \mathbb{E}\left(s_{k}\right) \mathbf{e}_{k}
$$

the covariance of

$$
\mathbf{\Upsilon}_{i} \triangleq \mathbb{C}\left(\mathbf{v}_{i}\right)=\sum_{k \neq i} \mathbb{C}\left(s_{k}\right) \mathbf{e}_{k} \mathbf{e}_{k}^{T}+2 \sigma^{2}\left(\mathbf{H}^{H} \mathbf{H}\right)^{-1},
$$

and the pseudo-covariance of

$$
\underline{\Upsilon}_{i} \triangleq \mathbb{C}_{p}\left(\mathbf{v}_{i}\right)=\sum_{k \neq i} \mathbb{C}_{p}\left(s_{k}\right) \mathbf{e}_{k} \mathbf{e}_{k}^{T}
$$

Note that (7) holds, because $\tilde{\mathbf{n}}$ is a circularly symmetric

\footnotetext{
${ }^{4}$ From another point of view, for a given data symbol $s_{k}$, the corresponding element of $\mathbf{v}_{i}$ has a Gaussian PDF centered around $s_{k}$. However, $s_{k}$ itself is a non-Gaussian random variable, which leads to a random displacement of the Gaussian PDF. As such, the accuracy of the Gaussian assumption for $\mathbf{v}_{i}$ does not improve, when $N_{t}$ or $N_{r}$ is increased.

${ }^{5} \mathrm{~A}$ proper complex random variable's pseudo-covariance vanishes [20], [21], and it is sufficient to characterize a proper complex Gaussian distribution using the mean and the covariance only. However, for a coded system using $M$-ary modulation, the in-phase and quadrature components of the complex modulated signal $s_{i}$ might be correlated and/or have different variances. In this case, it is necessary to take into account an additional secondorder statistics, i.e. the pseudo-covariance [20], to fully specify the improper complex Gaussian distribution in a generalized manner.
}

complex-valued Gaussian noise vector and hence it is proper [20], [21].

Considering the IDD architecture, we define an $\left(N_{t} \times M\right)$ element probability matrix $\mathbf{P}^{\left(z, z^{\prime}\right)}$, whose element $P_{n, m}^{\left(z, z^{\prime}\right)}$ represents the estimate of the APP that we have $s_{n}=a_{m}$ at the $z$-th $/ z^{\prime}$-th outer/inner iteration of the EB-Log-PDA approach. More precisely, we have

$$
P_{n, m}^{\left(z, z^{\prime}\right)}=P_{m}^{\left(z, z^{\prime}\right)}\left(s_{n} \mid \tilde{\mathbf{y}}\right) \triangleq P^{\left(z, z^{\prime}\right)}\left(s_{n}=a_{m} \mid \tilde{\mathbf{y}}\right),
$$

where $z$ and $z^{\prime}$ are nonnegative integers, $n=1, \cdots, N_{t}$ and $m=1, \cdots, M$. Then we have

$$
\begin{gathered}
\mathbb{E}\left(s_{k}\right)=\sum_{m=1}^{M} a_{m} P_{m}^{\left(z, z^{\prime}\right)}\left(s_{k} \mid \tilde{\mathbf{y}}\right), \\
\mathbb{C}\left(s_{k}\right)=\sum_{m=1}^{M}\left(a_{m}-\mathbb{E}\left(s_{k}\right)\right)\left(a_{m}-\mathbb{E}\left(s_{k}\right)\right)^{*} P_{m}^{\left(z, z^{\prime}\right)}\left(s_{k} \mid \tilde{\mathbf{y}}\right),
\end{gathered}
$$

and

$$
\mathbb{C}_{p}\left(s_{k}\right)=\sum_{m=1}^{M}\left(a_{m}-\mathbb{E}\left(s_{k}\right)\right)^{2} P_{m}^{\left(z, z^{\prime}\right)}\left(s_{k} \mid \tilde{\mathbf{y}}\right),
$$

for (5), (6) and (7), respectively, where the pseudo-covariance of a complex random vector $\mathbf{x}$ is defined as [20], [21]

$$
\mathbb{C}_{p}(\mathbf{x}) \triangleq \mathbb{E}\left[(\mathbf{x}-\mathbb{E}(\mathbf{x}))(\mathbf{x}-\mathbb{E}(\mathbf{x}))^{T}\right]
$$

Note that from Eq. (5) to Eq. (11) we effectively use $\left(N_{t}-\right.$ 1) probability vectors of $\left\{\mathbf{P}^{\left(z, z^{\prime}\right)}(k,:)\right\}_{k \neq i}$ associated with the interference signal $\left\{s_{k}\right\}_{k \neq i}$ to model $\mathbf{v}_{i}$, where $\mathbf{P}^{\left(z, z^{\prime}\right)}(k,:)$ represents the $k$ th row of the matrix $\mathbf{P}^{\left(z, z^{\prime}\right)}$. Since we do not have any outer a priori knowledge about the distribution of $s_{n} \mid \tilde{\mathbf{y}}$ at the beginning, $P_{m}^{\left(z, z^{\prime}\right)}\left(s_{n} \mid \tilde{\mathbf{y}}\right)$ is initialized using the uniform distribution of

$$
P_{m}^{(0,0)}\left(s_{n} \mid \tilde{\mathbf{y}}\right)=\frac{1}{M}
$$

for $\forall n=1, \cdots, N_{t}$ and $\forall m=1, \cdots, M$.

Based on the assumption that $\mathbf{v}_{i}$ obeys the Gaussian distribution, $\tilde{\mathbf{y}} \mid s_{i}$ is also Gaussian distributed. We define

$$
\mathbf{w} \triangleq \tilde{\mathbf{y}}-s_{i} \mathbf{e}_{i}-\sum_{k \neq i} \mathbb{E}\left(s_{k}\right) \mathbf{e}_{k}
$$

and

$$
\alpha_{i, m}^{\left(z, z^{\prime}+1\right)} \triangleq-\left[\begin{array}{c}
\Re(\mathbf{w}) \\
\Im(\mathbf{w})
\end{array}\right]^{T} \boldsymbol{\Lambda}_{i}^{-1}\left[\begin{array}{c}
\Re(\mathbf{w}) \\
\Im(\mathbf{w})
\end{array}\right],
$$

where the composite covariance matrix $\boldsymbol{\Lambda}_{i}$ is defined as

$$
\boldsymbol{\Lambda}_{i} \triangleq\left[\begin{array}{cc}
\Re\left(\boldsymbol{\Upsilon}_{i}+\underline{\Upsilon}_{i}\right) & -\Im\left(\Upsilon_{i}-\underline{\Upsilon}_{i}\right) \\
\Im\left(\mathbf{\Upsilon}_{i}+\underline{\Upsilon}_{i}\right) & \Re\left(\mathbf{\Upsilon}_{i}-\underline{\Upsilon}_{i}\right)
\end{array}\right]
$$

where $\Re(\cdot)$ and $\Im(\cdot)$ represent the real and imaginary part of a complex variable, respectively. Then the likelihood function of $\tilde{\mathbf{y}} \mid s_{i}=a_{m}$ at the $\left(z, z^{\prime}+1\right)$-st iteration satisfies

$$
p_{m}^{\left(z, z^{\prime}+1\right)}\left(\tilde{\mathbf{y}} \mid s_{i}\right) \propto \exp \left(\alpha_{i, m}^{\left(z, z^{\prime}+1\right)}\right) .
$$

In the next step, the existing PDA methods employed in uncoded MIMO systems typically invoked an approximate form of the Bayesian theorem to estimate the symbol-wise 
APPs [4]-[6], [9], [10], which is

$$
\mathcal{P}_{m}^{\left(z, z^{\prime}+1\right)}\left(s_{i} \mid \tilde{\mathbf{y}}\right) \approx \frac{p_{m}^{\left(z, z^{\prime}+1\right)}\left(\tilde{\mathbf{y}} \mid s_{i}\right)}{\sum_{m=1}^{M} p_{m}^{\left(z, z^{\prime}+1\right)}\left(\tilde{\mathbf{y}} \mid s_{i}\right)} .
$$

However, we argue that (18) does not conform to the formal mathematical definition of the APP in Bayesian statistics, ${ }^{6}$ which is [22]

$$
P(\theta \mid X) \propto P(\theta) P(X \mid \theta),
$$

where the probability $P(\theta)$ is an a priori belief of the random variable $\theta$, and $P(X \mid \theta)$ is the likelihood of the observation $X$. Therefore, $\mathcal{P}_{m}^{\left(z, z^{\prime}+1\right)}\left(s_{i} \mid \tilde{\mathbf{y}}\right)$ calculated with the aid of (18) does not represent the true APPs, but instead the normalized likelihoods, which may be regarded as a type of nominal APPs. $^{7}$

To obtain the true APP of symbol $s_{i}$ at the $\left(z, z^{\prime}+1\right)$-st iteration of the PDA-aided IDD receiver, we advocate to use the exact form of the Bayesian theorem. Hence the true APP estimated at the output of the PDA method is given as

$$
\begin{aligned}
& P_{m}^{\left(z, z^{\prime}+1\right)}\left(s_{i} \mid \tilde{\mathbf{y}}\right) \\
= & \frac{p_{m}^{\left(z, z^{\prime}+1\right)}\left(\tilde{\mathbf{y}} \mid s_{i}\right) P_{m}^{\left(z, z^{\prime}\right)}\left(s_{i}\right)}{\sum_{m=1}^{M} p_{m}^{\left(z, z^{\prime}+1\right)}\left(\tilde{\mathbf{y}} \mid s_{i}\right) P_{m}^{\left(z, z^{\prime}\right)}\left(s_{i}\right)} \\
= & \frac{\exp \left(\beta_{i, m}^{\left(z, z^{\prime}+1\right)}-\gamma\right)}{\sum_{m=1}^{M} \exp \left(\beta_{i, m}^{\left(z, z^{\prime}+1\right)}-\gamma\right)},
\end{aligned}
$$

where $P_{m}^{\left(z, z^{\prime}\right)}\left(s_{i}\right)$ is the a priori probability generated from the extrinsic LLRs fed back by the soft FEC decoder, and $\beta_{i, m}^{\left(z, z^{\prime}+1\right)} \triangleq \alpha_{i, m}^{\left(z, z^{\prime}+1\right)}+\ln \left(P_{m}^{\left(z, z^{\prime}\right)}\left(s_{i}\right)\right)$. Note that $\gamma \triangleq$ $\max _{m=1, \cdots, M} \beta_{i, m}^{\left(z, z^{\prime}+1\right)}$ is introduced for further improving the numerical stability and accuracy. For the same reason, (20) is reformulated in the logarithmic domain as

$$
\begin{aligned}
& \psi_{i, m}^{\left(z, z^{\prime}+1\right)} \\
\triangleq & \ln \left(P_{m}^{\left(z, z^{\prime}+1\right)}\left(s_{i} \mid \tilde{\mathbf{y}}\right)\right) \\
= & \tilde{\beta}_{i, m}^{\left(z, z^{\prime}+1\right)}-\ln \left(\sum_{m=1}^{M} \exp \left(\tilde{\beta}_{i, m}^{\left(z, z^{\prime}+1\right)}\right)\right),
\end{aligned}
$$

where we have $\tilde{\beta}_{i, m}^{\left(z, z^{\prime}+1\right)} \triangleq \beta_{i, m}^{\left(z, z^{\prime}+1\right)}-\gamma$, and the second term of the right-hand-side expression may be computed by invoking the "Jacobian logarithm' of [13]. When invoking the Max-log approximation, (21) may be further simplified as

$$
\psi_{i, m}^{\left(z, z^{\prime}+1\right)} \approx \tilde{\beta}_{i, m}^{\left(z, z^{\prime}+1\right)}-\max _{m=1, \cdots, M} \tilde{\beta}_{i, m}^{\left(z, z^{\prime}+1\right)}=\tilde{\beta}_{i, m}^{\left(z, z^{\prime}+1\right)} .
$$

\footnotetext{
${ }^{6}$ For the sake of clarity, here we use $\mathcal{P}()$ to denote the symbol-wise probabilities estimated using the approximate Bayesian formula given by (18), while using $P()$ to represent ordinary probabilities otherwise.

${ }^{7}$ Note that these nominal APPs were invoked in the uncoded systems of [4][10] and in the coded system of [15] without imposing any notable problems. This is because the calculation of extrinsic LLRs was not required in those contexts.
}

As a result, the estimated symbol-wise APP of $s_{i}$ is given by

$$
P_{m}^{\left(z, z^{\prime}+1\right)}\left(s_{i} \mid \tilde{\mathbf{y}}\right) \approx e^{\psi_{i, m}^{\left(z, z^{\prime}+1\right)}},
$$

which will be used for replacing the corresponding element $P_{m}^{\left(z, z^{\prime}\right)}\left(s_{i} \mid \tilde{\mathbf{y}}\right)$ in the probability matrix $\mathbf{P}^{\left(z, z^{\prime}\right)}$. Based on these updated symbol-wise APPs, the procedure presented above may be repeated either in the next inner iteration within the PDA or in the next outer iteration exchanging extrinsic information between the FEC-decoder and the MIMO detector to obtain new estimates of the symbol-wise APPs.

For the sake of clarity, the EB-Log-PDA algorithm relying on the a priori soft information feedback gleaned from the outer FEC decoder is summarized in Table I.

\section{Extrinsic LLR CAlCUlation For EB-LOG-PDA}

\section{A. Method of Calculating the Extrinsic LLRs for EB-Log-PDA}

For ease of exposition, in the following we will denote the left-hand-side term of (18) and (20) as $\mathcal{P}\left(s_{i}=a_{m} \mid \mathbf{y}\right)$ and $P\left(s_{i}=a_{m} \mid \mathbf{y}\right)$, respectively. As a beneficial result of replacing (18) by (20), the extrinsic LLRs may be calculated according to the classic relationship by subtracting the $a$ priori LLRs from the a posteriori LLRs that are generated from the estimated symbol-wise APPs of the EB-Log-PDA detector, hence we have

$$
\begin{aligned}
L_{E}^{E B}\left(b_{i l} \mid \mathbf{y}\right)= & L_{D}\left(b_{i l} \mid \mathbf{y}\right)-L_{A}\left(b_{i l}\right) \\
= & \ln \frac{P\left(b_{i l}=+1 \mid \mathbf{y}\right)}{P\left(b_{i l}=-1 \mid \mathbf{y}\right)}-L_{A}\left(b_{i l}\right) \\
= & \ln \frac{\sum_{\forall a_{m} \in \mathcal{A}_{l}^{+}} P\left(s_{i}=a_{m} \mid \mathbf{y}\right)}{\sum_{\forall a_{m} \in \mathcal{A}_{l}^{-}} P\left(s_{i}=a_{m} \mid \mathbf{y}\right)} \\
& -\underbrace{\ln \frac{P\left(b_{i l}=+1\right)}{P\left(b_{i l}=-1\right)}}_{L_{A}\left(b_{i l}\right)},
\end{aligned}
$$

where $\mathcal{A}_{l}^{ \pm}$denotes the set of $M / 2$ constellation points whose $l$ th bit is +1 or -1 , respectively. It is noteworthy that (24) represents a simpler technique of generating the bit-wise extrinsic LLRs $L_{E}^{E B}\left(b_{i l} \mid \mathbf{y}\right)$, as long as the true symbol-wise APPs of $P\left(s_{i}=a_{m} \mid \mathbf{y}\right)$ may be obtained.

By contrast, this approach is not applicable to the conventional approximate Bayesian theorem based PDA methods [4]-[10]. Our study reveals that the estimated symbol-wise nominal APPs obtained from (18) are unsuitable for generating the correct extrinsic bit-wise LLRs upon invoking the classic formula (24). This hidden fact is corroborated by the simulation results of Fig. 2. In the scenarios where the number of outer iterations $i t_{o}$ was set to be higher than zero, it was observed in Fig. 2 that the BER results of the IDD scheme using the AB-Log-PDA and (24) became abnormally poor, when the nominal symbol-wise APPs produced by (18) were misinterpreted as true symbol-wise APPs. More specifically, we can observe from Fig. 2 that except for $i t_{o}=0$, the BER of the AB-Log-PDA based IDD scheme unexpectedly degrades upon increasing $E_{b} / N_{0}$, and it also deteriorates when $i t_{o}$ increases from 1 to 4 . The BER curve of the $i t_{o}=0$ scenario characterized in Fig. 2 exhibits a trend 
TABLE I

SUMMARY OF THE EB-LOG-PDA ALGORITHM

Given the received signal $\mathbf{y}$, the channel matrix $\mathbf{H}$ and the modulation constellation $\mathcal{A}$, make preprocessing on the received signal $\mathbf{y}$ to obtain $\tilde{\mathbf{y}}=\left(\mathbf{H}^{H} \mathbf{H}\right)^{-1} \mathbf{H}^{H} \mathbf{y}$. Step 1. Set the initial value of the inner iteration index to $z^{\prime}=0$. If the outer iteration index $z=0$, initialize the values of the symbol-wise APPs as $P_{m}^{\left(z, z^{\prime}\right)}\left(s_{n} \mid \tilde{\mathbf{y}}\right)=1 / M$, for $\forall n=1,2, \cdots, N_{t}$ and $\forall m=1,2, \cdots, M$. Otherwise, initialize the values of $P_{m}^{\left(z, z^{\prime}\right)}\left(s_{n} \mid \tilde{\mathbf{y}}\right)$ using the a priori probabilities generated from the feedback extrinsic LLRs of the soft FEC decoder. Step 2. Based on the values of $\left\{\mathbf{P}^{\left(z, z^{\prime}\right)}(k,:)\right\}_{k \neq i}$, calculate $P_{m}^{\left(z, z^{\prime}+1\right)}\left(s_{i} \mid \tilde{\mathbf{y}}\right)$ : for $i=1: N_{t}$

calculate the statistics of the interference-plus-noise term $\mathbf{v}_{i}$ using (5) - (11), as well as the inverse of $\boldsymbol{\Lambda}_{i}$ in (16),

for $m=1: M$

calculate $P_{m}^{\left(z, z^{\prime}+1\right)}\left(s_{i} \mid \tilde{\mathbf{y}}\right)$ using (14), (15), (21) and (23).

end

end

Step 3. If $\left|P_{m}^{\left(z, z^{\prime}+1\right)}\left(s_{i} \mid \tilde{\mathbf{y}}\right)-P_{m}^{\left(z, z^{\prime}\right)}\left(s_{i} \mid \tilde{\mathbf{y}}\right)\right|<\varepsilon, \forall i$ and $\forall m$, i.e. the probability-matrix $\mathbf{P}^{\left(z, z^{\prime}+1\right)}$ has converged, where $\varepsilon$ is a given small positive real number, or the index $z^{\prime}$ has reached a given number of iterations, terminate the iteration and output $\mathbf{P}^{\left(z, z^{\prime}+1\right)}$. Otherwise, let $z^{\prime}=z^{\prime}+1$ and return to Step 2.

in line with our expectations, because in this case no soft information is fed back from the FEC decoder and hence the term $P_{m}^{\left(z, z^{\prime}\right)}\left(s_{i}\right)$ in $(20)$ can be eliminated. In other words, (18) becomes equivalent to (20) in this scenario. These results further demonstrate that (18) should be regarded as a sort of symbol-wise nominal APP, rather than the symbol-wise true APP, as calculated in (20). As a result, we argue that in order to generate the correct symbol-wise APPs, which are readily applicable to (24), the calculation of $P_{m}^{\left(z, z^{\prime}+1\right)}\left(s_{i} \mid \tilde{\mathbf{y}}\right)$ has to rely on (20) instead of (18) in the PDA based MIMO detector.

Slightly differently from the above-mentioned $M$-ary modulation scenarios, there exist two alternative methods of generating the extrinsic bit-wise LLRs for BPSK modulation aided MIMO systems. On the one hand, the extrinsic LLR of $b_{i l}$, simplified as $b_{i}$, may be formulated as

$$
\begin{aligned}
L_{E}^{E B}\left(b_{i} \mid \mathbf{y}\right) & =L_{D}\left(b_{i} \mid \mathbf{y}\right)-L_{A}\left(b_{i}\right) \\
& =\ln \frac{P\left(b_{i}=+1 \mid \mathbf{y}\right)}{P\left(b_{i}=-1 \mid \mathbf{y}\right)}-\ln \frac{P\left(b_{i}=+1\right)}{P\left(b_{i}=-1\right)}
\end{aligned}
$$

where $P\left(b_{i}= \pm 1 \mid \mathbf{y}\right)$ is the final output of the EB-Log-PDA algorithm for BPSK modulation. Alternatively, we have

$$
L_{D}\left(b_{i} \mid \mathbf{y}\right)=\ln \frac{p\left(\mathbf{y} \mid b_{i}=+1\right)}{p\left(\mathbf{y} \mid b_{i}=-1\right)}+\ln \frac{P\left(b_{i}=+1\right)}{P\left(b_{i}=-1\right)} .
$$

Since $p\left(\mathbf{y} \mid b_{i}= \pm 1\right)$ constitutes the intermediate output of the EB-Log-PDA algorithm for BPSK modulation, we can directly obtain

$$
L_{E}^{E B}\left(b_{i} \mid \mathbf{y}\right)=\ln \frac{p\left(\mathbf{y} \mid b_{i}=+1\right)}{p\left(\mathbf{y} \mid b_{i}=-1\right)} .
$$

\section{B. On the Relationship Between the Extrinsic LLRs of the EB- Log-PDA and AB-Log-PDA}

According to Conjecture 1 of [16], [17], the bit-wise extrinsic LLRs of the AB-Log-PDA algorithm relying on (18)

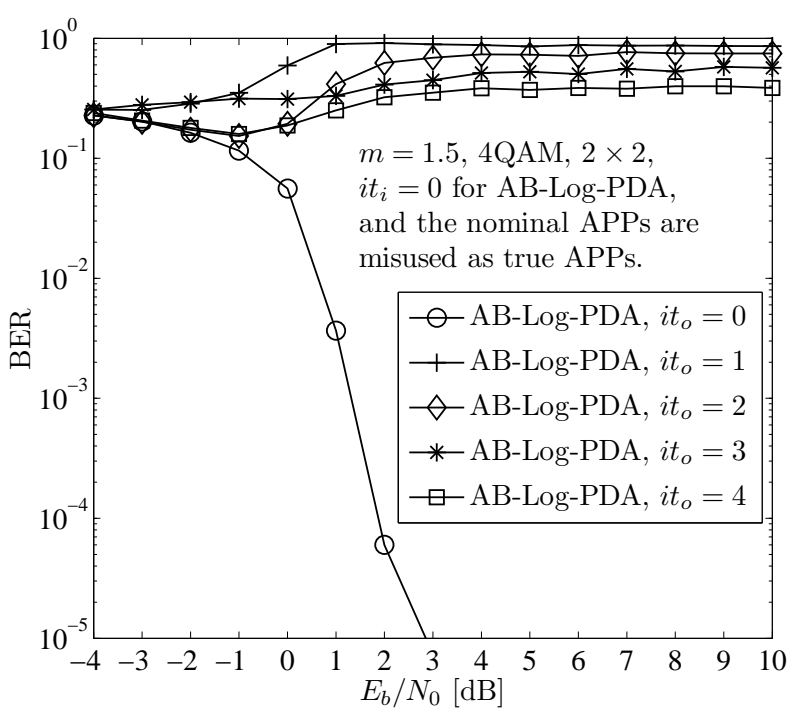

Fig. 2. BER of the AB-Log-PDA based IDD scheme, where the nominal symbol-wise APPs were misinterpreted as the true symbol-wise APPs.

may be approximated by

$$
L_{E}^{A B}\left(b_{i l} \mid \mathbf{y}\right) \approx \ln \frac{\sum_{\forall a_{m} \in \mathcal{A}_{l}^{+}} \mathcal{P}\left(s_{i}=a_{m} \mid \mathbf{y}\right)}{\sum_{\forall a_{m} \in \mathcal{A}_{l}^{-}} \mathcal{P}\left(s_{i}=a_{m} \mid \mathbf{y}\right)},
$$

where $\mathcal{P}\left(s_{i}=a_{m} \mid \mathbf{y}\right)$ is calculated by invoking (18). The resultant $L_{E}^{A B}\left(b_{i l} \mid \mathbf{y}\right)$ in (28) was shown to work well in the AB-Log-PDA aided IDD scheme of [16], [17], although the right-hand-side expression of (28) appears to be in the form of $L_{D}^{A B}\left(b_{i l} \mid \mathbf{y}\right)$ compared with (24). Therefore, it is important to investigate the relationship between the extrinsic LLRs of the EB-Log-PDA formulated in (24) and that of the AB-Log-PDA calculated by (28).

Theorem 1. For a specific bit $b_{i l}$, the extrinsic LLR of the $E B-L o g-P D A$ algorithm is typically different from that of the AB-Log-PDA algorithm.

Proof: Based on $b_{i l}=+1$ or -1 , the symbols set $\mathcal{A}$ may be divided into the pair of subsets $\mathcal{A}_{l}^{+}$and $\mathcal{A}_{l}^{-}$, each hosting half of the constellation symbols. Without loss of generality, we assume that $\mathcal{A}_{l}^{+}=\left\{a_{1}, a_{2}, \cdots, a_{M / 2}\right\}$, and $\mathcal{A}_{l}^{-}=\left\{a_{M / 2+1}, a_{M / 2+2}, \cdots, a_{M}\right\}$. By substituting (18) into (28), we arrive at

$$
\begin{aligned}
& L_{E}^{A B}\left(b_{i l} \mid \mathbf{y}\right) \\
= & \ln \frac{\left(\sum_{\forall a_{m} \in \mathcal{A}_{l}^{+}} p\left(\mathbf{y} \mid s_{i}=a_{m}\right)\right) /\left(\sum_{m=1}^{M} p\left(\mathbf{y} \mid s_{i}=a_{m}\right)\right)}{\left(\sum_{\forall a_{m} \in \mathcal{A}_{l}^{-}} p\left(\mathbf{y} \mid s_{i}=a_{m}\right)\right) /\left(\sum_{m=1}^{M} p\left(\mathbf{y} \mid s_{i}=a_{m}\right)\right)} \\
= & \ln \frac{\sum_{\forall a_{m} \in \mathcal{A}_{l}^{+}} p\left(\mathbf{y} \mid s_{i}=a_{m}\right)}{\sum_{\forall a_{m} \in \mathcal{A}_{l}^{-}} p\left(\mathbf{y} \mid s_{i}=a_{m}\right)} .
\end{aligned}
$$


On the other hand, by substituting (20) into (24), we have

$$
\begin{aligned}
& L_{E}^{E B}\left(b_{i l} \mid \mathbf{y}\right)=\ln \frac{\sum_{\forall a_{m} \in \mathcal{A}_{l}^{+}} p\left(\mathbf{y} \mid s_{i}=a_{m}\right) P\left(s_{i}=a_{m}\right)}{\sum_{\forall a_{m} \in \mathcal{A}_{l}^{-}} p\left(\mathbf{y} \mid s_{i}=a_{m}\right) P\left(s_{i}=a_{m}\right)} \\
& -\ln \frac{P\left(b_{i l}=+1\right)}{P\left(b_{i l}=-1\right)} \\
& =\ln \frac{\sum_{\forall a_{m} \in \mathcal{A}_{l}^{+}} p\left(\mathbf{y} \mid s_{i}=a_{m}\right) P\left(s_{i}=a_{m}\right)}{\sum_{\forall a_{m} \in \mathcal{A}_{l}^{-}} p\left(\mathbf{y} \mid s_{i}=a_{m}\right) P\left(s_{i}=a_{m}\right)} \\
& -\ln \frac{\sum_{\forall a_{m} \in \mathcal{A}_{l}^{+}} P\left(s_{i}=a_{m}\right)}{\sum_{\forall a_{m} \in \mathcal{A}_{l}^{-}} P\left(s_{i}=a_{m}\right)} .
\end{aligned}
$$

Note that (29) and (30) are typically not equal to each other, because i) the term $p\left(\mathbf{y} \mid s_{i}=a_{m}\right)$ in (29) and in (30) might be different if the number of inner iterations within the PDAs is higher than zero ${ }^{8}$;ii) even if the term $p\left(\mathbf{y} \mid s_{i}=a_{m}\right)$ in (29) and in (30) would be equal to each other at the same iteration index $\left(z, z^{\prime}\right)$, typically (29) and (30) would still be different from each other, except in some specific circumstances, for example, when $P\left(s_{i}=a_{m}\right)$ in (30) has the same value for the different constellation symbols $a_{m}$.

Apart from the nature of difference between the two types of extrinsic LLRs encapsulated in Theorem 1, the following proposition further characterizes the degree of difference between the two types of extrinsic LLRs.

Proposition 1. Let us consider the difference $\Delta L_{i l}=$ $L_{E}^{E B}(i, l)-L_{E}^{A B}(i, l)$ between the two types of extrinsic LLRs for each specific bit $b_{i l}$, and also the difference between the hard-decision results $\operatorname{sgn}\left[L_{E}^{E B}(i, l)\right]$ and $\operatorname{sgn}\left[L_{E}^{A B}(i, l)\right]$ based on the two types of extrinsic LLRs for each specific bit $b_{i l}$. Then, the bit-wise extrinsic LLRs $L_{E}^{E B}(i, l)$ of the EB-Log$P D A$ and $L_{E}^{A B}(i, l)$ of the AB-Log-PDA are found to be statistically closest to each other, when the mutual information (MI) between the bit-sequence output by the interleaver and the corresponding a priori LLRs fed back by the outer FEC decoder, namely $I_{A}\left(\mathbf{d}_{2}, \mathbf{L}_{A_{2}}\right)$, approaches either its minimum value of 0.0 or its maximum value of 1.0.

\section{Remarks:}

1) This proposition is inferred from the statistical results shown in Table II, Fig. 9 and Fig. 10. It can also be verified by comparing (29) and (30). More specifically, in the first case, if we have no a priori information about the bit-sequence output by the interleaver, then $I_{A}\left(\mathbf{d}_{2}, \mathbf{L}_{A_{2}}\right)$ has a minimum value of 0.0 , and $P\left(s_{i}=\right.$ $\left.a_{m}\right)$ in (30) becomes a constant for all $a_{m}$. Hence (30) may be simplified to the form of (29). Similarly, in the second case, when the reliability of the a priori LLRs fed back by the outer FEC decoder becomes sufficiently high, namely $I_{A}\left(\mathbf{d}_{2}, \mathbf{L}_{A_{2}}\right)$ approaches its

\footnotetext{
${ }^{8}$ The estimated symbol-wise probabilities which are used for calculating the statistics of the interference-plus-noise term $\mathbf{v}_{i}$ via (5) - (11) might be different for the AB-Log-PDA and EB-Log-PDA algorithms, even if the iteration index $\left(z, z^{\prime}\right)$ with $z^{\prime}>0$ is the same for them.
}

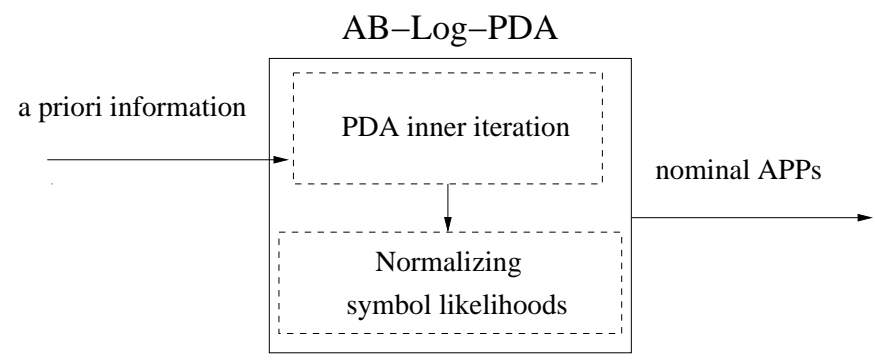

(a)

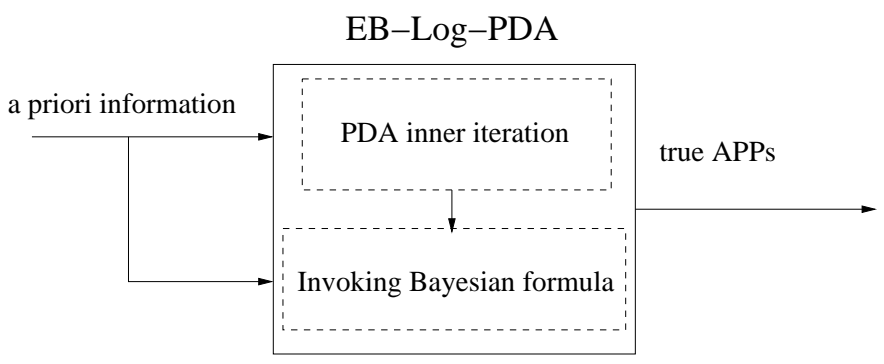

(b)

Fig. 3. The role of the a priori information in the AB-Log-PDA and the EB-Log-PDA algorithms.

maximum value of 1.0, then there exists a single dominant probability which approaches 1.0 among the $a$ priori symbol-wise probabilities $P\left(s_{i}=a_{m}\right)$ for all $a_{m}$. In this case, (30) can also be approximated by the form of (29), albeit due to the associated approximation, the number of identical pairs of the two types of extrinsic LLRs becomes lower than that in the first case, as shown in the second column of Table II.

2) As shown in Fig. 3 and (18), the AB-Log-PDA has already invoked the a priori information as the input of the PDA's inner iteration for generating the symbolwise nominal APPs. By comparison, the EB-Log-PDA invokes the same a priori information twice: one for the PDA's inner iteration (similar to the case of ABLog-PDA), and the other for invoking the Bayesian formula. In other words, the same a priori information is invoked twice for providing different functions in two different processing blocks. This feature is also distinct from the case of the MAP-based MIMO detection, where the a priori information is used once and it is uncorrelated with the function of generating the symbolvector likelihoods $p(\mathbf{y} \mid \mathbf{s})$.

\section{Simulation Results and Discussions}

In this section, we characterize both the convergence behavior and the achievable performance of the proposed EBLog-PDA based IDD scheme with the aid of both the semianalytical extrinsic information transfer (EXIT) charts [23] and Monte-Carlo simulations. Furthermore, we investigate the impact of inner PDA iterations on the attainable performance of both the EB-Log-PDA and the AB-Log-PDA based IDD schemes, as well as the relationship between the extrinsic LLRs of the EB-Log-PDA and the AB-Log-PDA. Additionally, the complexity of the proposed EB-Log-PDA based IDD scheme is compared both to that of the AB-Log- 
PDA and to that of the optimal MAP based IDD schemes, which demonstrates the attractive performance versus complexity tradeoff achieved by the proposed EB-Log-PDA based IDD scheme. The turbo code employed uses two constituent recursive systematic convolutional (RSC) codes concatenated in parallel [24]. The RSC codes have a constraint length of $L=3$ and generator polynomials of $(7,5)$ expressed in octal form, where half of the parity bits generated by each of the two RSC codes are punctured, so that the turbo code employed has a coding rate of $R=\frac{k}{n}=1 / 2$. The turbo code is decoded by the Approximate-Log-MAP algorithm using $i t_{t c}=4$ inner iterations. The interleaver employed is a 2400 bit random sequence interleaver. The remaining scenariodependent simulation parameters are shown in the respective figures, where the MIMO arrangement is represented in the form of $\left(N_{t} \times N_{r}\right)$.

\section{A. Convergence and Performance of the EB-Log-PDA based IDD}

Fig. 4 compares the convergence behavior of both the proposed EB-Log-PDA based IDD, as well as of the ABLog-PDA based IDD and of the optimal Exact-Log-MAP based IDD schemes using EXIT chart [23] analysis. It is observed that the EXIT curve of the EB-Log-PDA is close to that of the Exact-Log-MAP, and almost overlaps with that of the AB-Log-PDA. More particularly, when the a priori MI is $I_{A, \text { inner }}=0$, the Exact-Log-MAP has the highest extrinsic MI of $I_{E \text {,outer }}=0.5596$, while the EB-Log-PDA has a higher extrinsic MI than the the AB-Log-PDA, which is $I_{E, \text { outer }}=0.5334$ versus $I_{E, \text { outer }}=0.5332$. This indicates that the achievable performances of the EB-Log-PDA and of the AB-Log-PDA are similar to each other, and both of them are close to that of the optimal Exact-Log-MAP in the scenario considered. Additionally, the Monte-Carlo simulation based detection/decoding trajectories indicate that the EBLog-PDA, the AB-Log-PDA and the Exact-Log-MAP based IDD schemes converge after three iterations. Furthermore, the performance improvements achieved at each iteration by the EB-Log-PDA are more significant than those of the AB-LogPDA, but less significant than those of the Exact-Log-MAP.

The above EXIT chart based performance predictions and the convergence behavior of the IDD schemes considered are also characterized in terms of the BER performance results of Fig. 5, where the Nakagami- $m$ fading parameter is set to $m=1.5$. Observe from Fig. 5 that the performance of the EBLog-PDA based IDD scheme is improved upon increasing the number of outer iterations $i t_{o}$, where $i t_{o}=0$ represents the conventional receiver structure in which the MIMO detector and the FEC decoder are serially concatenated, but operate without exchanging soft information. However, the attainable improvements become gradually smaller and the performance achieved after three outer iterations in Fig. 5 becomes similar to that of four outer iterations. This implies that the EB-LogPDA based IDD scheme essentially converges after three outer iterations. A similar convergence profile is also observed in Fig. 5 for the optimal Exact-Log-MAP based IDD, although its performance is always marginally better than that of the corresponding EB-Log-PDA based IDD.

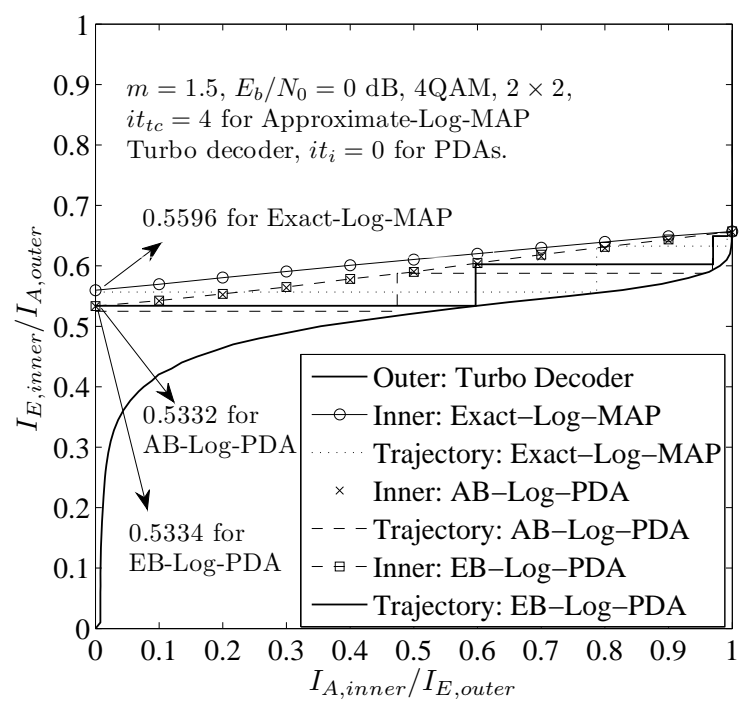

Fig. 4. EXIT chart analysis of the EB-Log-PDA, AB-Log-PDA and ExactLog-MAP based IDD schemes.

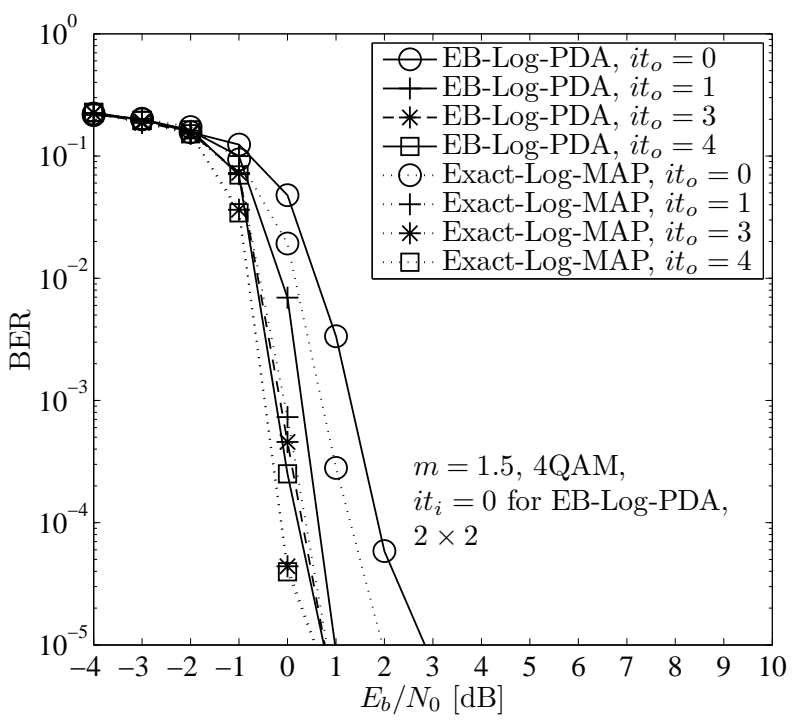

Fig. 5. Impact of the number of outer iterations on BER of the EB-Log-PDA based IDD scheme.

\section{B. Comparison of the Impact of Inner PDA Iterations on the Performance of the Two Types of PDA-based IDDs}

In Fig. 6, we investigate the impact of the number of inner iterations of the EB-Log-PDA on the achievable performance of the IDD scheme. We observe that the performance of the IDD scheme is degraded as the number of its inner iterations increases, despite its increased computational complexity. ${ }^{9}$ This implies that the optimal number of inner iterations of the EB-Log-PDA conceived for the IDD receiver is $i t_{i}=z^{\prime}=0$. This is because the inner PDA iterations typically fail to assist the repeated Gaussian approximation process in finding the global optimum [9]. Hence, when the reliability of the soft

\footnotetext{
${ }^{9}$ This observation was found to be also valid for other system configurations, for example, for $N_{t}=N_{r}=4$ and $i t_{o}=2$. However, due to page limitations, these numerical results are not provided here.
} 


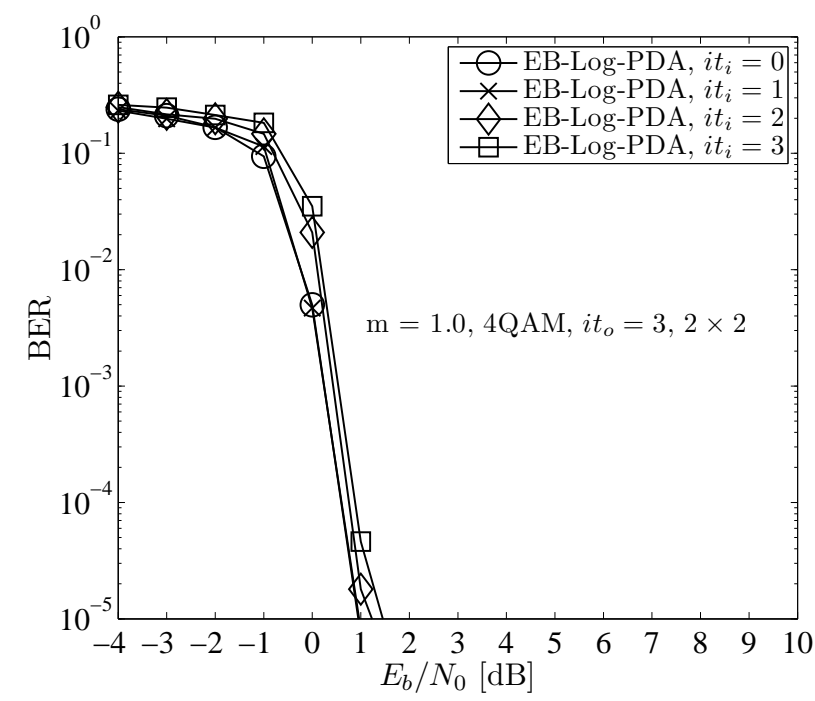

Fig. 6. Impact of the number of inner iterations within the EB-Log-PDA on BER of the EB-Log-PDA based IDD scheme.

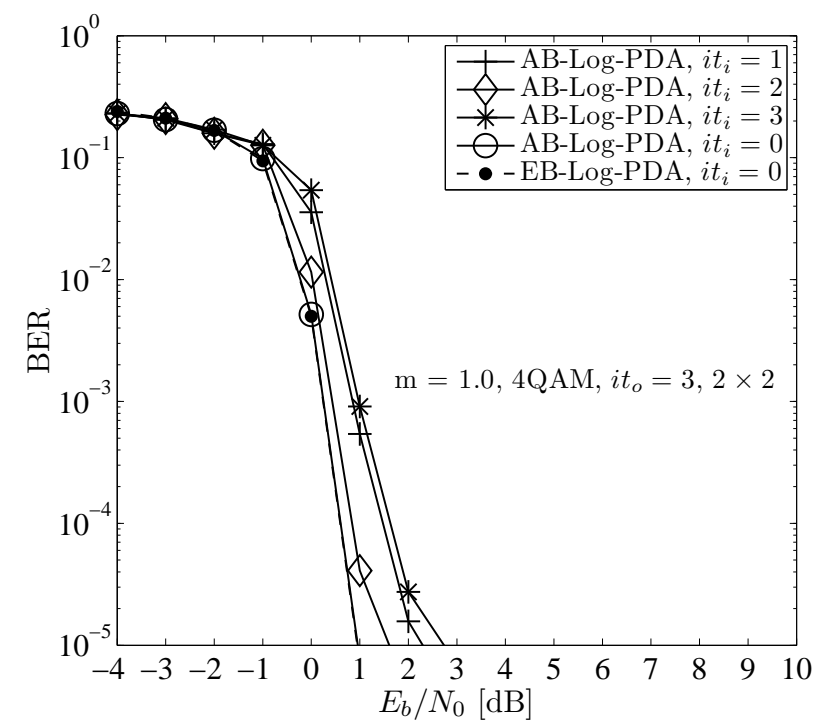

Fig. 7. Impact of the number of inner PDA iterations on BER of the ABLog-PDA based IDD scheme, as well as the BER comparison between the EB-Log-PDA and AB-Log-PDA based IDD schemes with $i t_{o}=3$.

information fed back from the FEC decoder is beyond the reliability limit that the inner iteration aided PDA can achieve, the better-quality feedback of the FEC decoder tends to be degraded towards this limit.

In Fig. 7 the impact of the number of inner AB-Log-PDA iterations on the achievable performance of the IDD scheme is investigated. More specifically, it is observed from Fig. 7 that the optimal number of inner PDA iterations of the AB-LogPDA based IDD is also $i t_{i}=0$, but the specific performance degradation experienced upon increasing the number of inner AB-Log-PDA iterations is slightly different from that of the EB-Log-PDA based IDD, as shown in Fig. 6. This indicates that the numerical stability of the AB-Log-PDA based IDD scheme incorporating inner PDA iterations is not as good as that of its counterpart using the EB-Log-PDA. Additionally, it is shown in Fig. 7 that the best achievable performance of the AB-Log-PDA based IDD scheme using $i t_{i}=0$ is almost identical to that of the EB-Log-PDA based IDD scheme.

Additionally, in Fig. 8 we investigate the intermediate BER evaluated at the output of the soft MIMO detectors, namely the BER recorded upon applying hard-decision to $\mathbf{L}_{D_{2}}$ for recovering the bits in $\mathbf{d}_{2}$ as shown in Fig. 1, when the soft MIMO detector employed is the EB-Log-PDA and AB-LogPDA, respectively ${ }^{10}$. It is observed that the EB-Log-PDA has a better intermediate BER performance than the AB-Log-PDA. This is because $\mathbf{L}_{D_{2}}$ represents the true a posteriori LLR for the EB-Log-PDA, whereas $\mathbf{L}_{D_{2}}$ is actually its extrinsic LLR generated in (28) for the AB-Log-PDA, where the nominal symbol-wise APPs are used.

By jointly considering the results of Fig. 6 - Fig. 8, we conclude that it is not recommended to incorporate inner iterations into the PDA algorithms, when designing the PDAbased IDD schemes.

\section{The Relationship Between the Extrinsic LLRs of the EB- Log-PDA and the AB-Log-PDA}

In Fig. 9, the CDFs of the difference between the two types of extrinsic LLRs, defined as $\Delta L_{i l}=L_{E}^{E B}(i, l)-L_{E}^{A B}(i, l)$, is presented for scenarios associated with different values of $I_{A}\left(\mathbf{d}_{2}, \mathbf{L}_{A_{2}}\right)$, which represents the MI between the bitsequence output by the interleaver and the corresponding a priori LLRs fed back by the outer FEC decoder. For convenience, $I_{A}\left(\mathbf{d}_{2}, \mathbf{L}_{A_{2}}\right)$ is simply denoted as $I_{A}$ in Fig. 9 and Table II. Observed in Fig. 9 that the CDFs may be divided into two groups of functions exhibiting rotational symmetry with respect to the point of $(0,0.5)$. The first group represents the CDFs associated with the $I_{A}$ value spanning from 0 to 0.6 , while the other group hosts the CDFs associated with the $I_{A}$ value ranging from 0.7 to 1.0. Interestingly, the two groups of CDFs exhibit opposite trends, when increasing the value of $I_{A}$. More specifically, upon considering the CDF curves falling within the right half-plane, we notice that when $I_{A}$ increases from 0 to 0.6 with a step size of 0.1 , the $\mathrm{CDF}$ curves of the first group move along from the point $(0,1)$ to its rightdownwards direction. By contrast, when $I_{A}$ increases from 0.7 to 1.0 with the step size of 0.1 , the CDF curves of the second group move along in the opposite direction compared to the first group. Additionally, by zooming in for observing the curve of $I_{A}=0.6$ and $I_{A}=0.7$, we note that the two curves almost overlap with each other and hence they serve as the boundary between the two groups. In line with Proposition 1 , the above observations clearly show that the bit-wise extrinsic LLRs of the EB-Log-PDA and of the AB-Log-PDA are statistically closest to each other, when $I_{A}$ approaches either its minimum value of 0.0 or its maximum value of 1.0 , and the difference between the two types of extrinsic LLRs becomes most significant, when the value of $I_{A}$ is in the middle region, say, between 0.4 to 0.7 . These conclusions are also confirmed by the corresponding PDFs shown in Fig. 10, where both the "linear scale" and the "semilogy" scale" are

\footnotetext{
${ }^{10}$ Note that this intermediate BER is different from the BER in uncoded systems, because the outer iterations also contribute to the output of the sof MIMO detectors.

${ }^{11}$ This means only the $y$-axis is in logarithmic scale.
} 
TABLE II

StATISTICAL COMPARISON OF $\mathbf{L}_{E}^{E B}$ AND $\mathbf{L}_{E}^{A B}$ FOR SAMPLES OF 240,000 BITS, $i t_{i}=0$ INNER PDA ITERATION, AND $m=1.0$.

\begin{tabular}{c|c|c|c|c|c|c|c|c|c|c}
\hline$I_{A}$ & $\begin{array}{c}\# \text { of } \\
\Delta L_{i}=0\end{array}$ & $\min \Delta L_{i}$ & $\max \Delta L_{i}$ & $\min \left|\Delta L_{i}\right|$ & $\max \left|\Delta L_{i}\right|$ & $\operatorname{mean}\left(\left|\Delta L_{i}\right|\right)$ & $\operatorname{mean}\left(\Delta L_{i}\right)$ & $\operatorname{var}\left(\left|\Delta L_{i}\right|\right)$ & $\operatorname{var}\left(\Delta L_{i}\right)$ & $\begin{array}{c}\# \operatorname{of} \operatorname{sgn}\left(L_{E}^{E B}(i)\right) \\
\neq \operatorname{sgn}\left(L_{E}^{A B}(i)\right)\end{array}$ \\
\hline 0 & 132564 & $-5.3291 \mathrm{e}-15$ & $7.1054 \mathrm{e}-15$ & 0 & $7.1054 \mathrm{e}-15$ & $2.1777 \mathrm{e}-16$ & $1.2659 \mathrm{e}-18$ & $1.0895 \mathrm{e}-31$ & $1.5637 \mathrm{e}-31$ & 0 \\
\hline 0.1 & 0 & -0.7602 & 0.5945 & $1.7764 \mathrm{e}-15$ & 0.7602 & 0.0029 & $-1.0932 \mathrm{e}-05$ & $1.0259 \mathrm{e}-04$ & $1.1092 \mathrm{e}-04$ & 93 \\
\hline 0.2 & 1 & -1.2742 & 1.5731 & 0 & 1.5731 & 0.0087 & $1.1815 \mathrm{e}-04$ & $8.2980 \mathrm{e}-04$ & $9.0512 \mathrm{e}-04$ & 236 \\
\hline 0.3 & 0 & -1.8188 & 1.9493 & $1.5099 \mathrm{e}-14$ & 1.9493 & 0.0159 & $5.9897 \mathrm{e}-05$ & 0.0025 & 0.0027 & 453 \\
\hline 0.4 & 0 & -2.1574 & 2.8015 & $2.6645 \mathrm{e}-15$ & 2.8015 & 0.0231 & $3.7206 \mathrm{e}-05$ & 0.0049 & 0.0055 & 575 \\
\hline 0.5 & 1 & -2.7502 & 3.7835 & 0 & 3.7835 & 0.0296 & $-6.2531 \mathrm{e}-05$ & 0.0080 & 0.0089 & 738 \\
\hline 0.6 & 0 & -3.8543 & 3.6252 & $3.5527 \mathrm{e}-15$ & 3.8543 & 0.0339 & $-2.1830 \mathrm{e}-05$ & 0.0113 & 0.0124 & 859 \\
\hline 0.7 & 0 & -3.4085 & 3.1513 & $5.3291 \mathrm{e}-15$ & 3.4085 & 0.0344 & $-3.9238 \mathrm{e}-05$ & 0.0132 & 0.0144 & 794 \\
\hline 0.8 & 0 & -3.4185 & 5.2725 & $3.5527 \mathrm{e}-15$ & 5.2725 & 0.0300 & $-8.3517 \mathrm{e}-05$ & 0.0131 & 0.0140 & 685 \\
\hline 0.9 & 3 & -4.6007 & 3.1890 & 0 & 4.6007 & 0.0190 & $8.8096 \mathrm{e}-06$ & 0.0088 & 0.0091 & 369 \\
\hline 1.0 & 6334 & $-3.3751 \mathrm{e}-14$ & $3.0198 \mathrm{e}-14$ & 0 & $3.3751 \mathrm{e}-14$ & $9.3921 \mathrm{e}-15$ & $-2.6836 \mathrm{e}-17$ & $4.4944 \mathrm{e}-29$ & $1.3315 \mathrm{e}-28$ & 0 \\
\hline
\end{tabular}

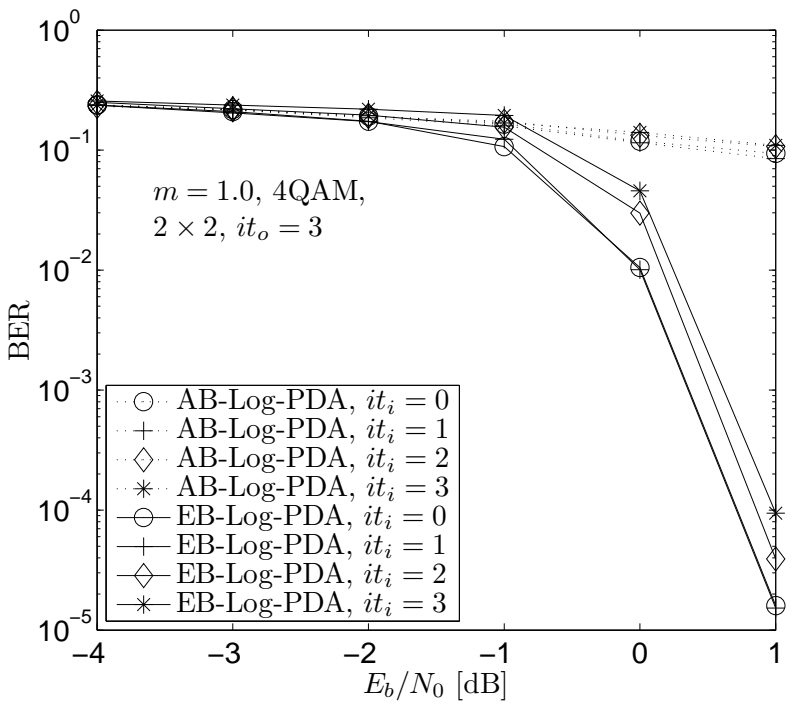

Fig. 8. Impact of the number of inner iterations on BER of the AB-Log-PDA and EB-Log-PDA MIMO detectors, evaluated using $\mathbf{L}_{D_{2}}$ at the output of the MIMO detectors for FEC-coded bits $\mathbf{d}_{2}$ in Fig. $1, i t_{o}=3$.

used for facilitating accurate observations. Finally, in Table II, the detailed statistical metrics of $\Delta L_{i l}=L_{E}^{E B}(i, l)-L_{E}^{A B}(i, l)$ are provided, which also support our conclusions.

\section{Complexity Comparison}

The computational complexity of the proposed EB-LogPDA based IDD scheme may be evaluated by simply comparing its complexity to those of the AB-Log-PDA and the Exact-Log-MAP based IDDs in a single (outer) iteration. This is because i) the turbo codec module is common to all the IDD schemes considered; 2) we have shown that the EB-LogPDA, as well as the AB-Log-PDA and the Exact-Log-MAP based IDD schemes all converge after three outer iterations in the scenarios considered; 3 ) the PDAs' inner iterations should not be invoked when designing IDD schemes. As shown in Table I, the major computational cost of the EB-Log-PDA per transmit symbol is the calculation of $\boldsymbol{\Lambda}_{i}^{-1}$ and the matrix multiplication of (15). Direct calculation of $\boldsymbol{\Lambda}_{i}^{-1}$ imposes a

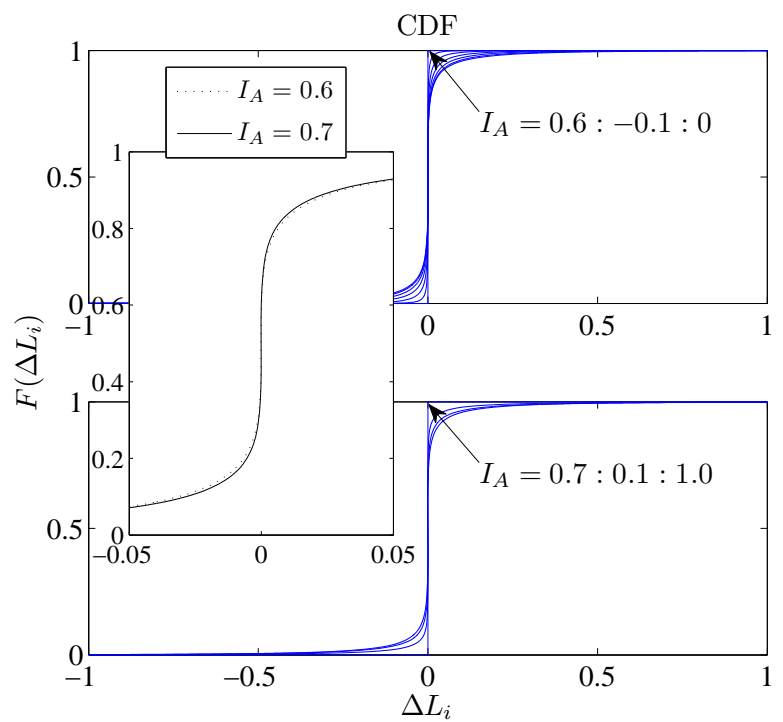

Fig. 9. The cumulative distribution functions of $\Delta L_{i}=L_{E}^{E B}(i)-L_{E}^{A B}(i)$, for samples of 240,000 bits, $i t_{i}=0$ inner PDA iteration, and $m=1.0$.

computational cost of $\mathcal{O}\left(8 N_{t}^{3}\right)$ real-valued operations (additions/multiplications), which is still relatively expensive. Fortunately, by using the Sherman-Morrison-Woodbury formula based complexity-reduction techniques of [4], the computational cost of calculating $\boldsymbol{\Lambda}_{i}^{-1}$ can be reduced to $\mathcal{O}\left(4 N_{t}^{3}\right)$ real operations per iteration. Additionally, the calculation of (15) requires $\mathcal{O}\left(4 M N_{t}^{3}+2 M N_{t}^{2}\right)$ real operations per iteration. In summary, in terms of real operations, the computational complexity of the decoupled signal model based EB-Log-PDA method is $\mathcal{O}\left(4 M N_{t}^{3}+2 M N_{t}^{2}\right)+\mathcal{O}\left(4 N_{t}^{3}\right)$ per outer iteration, which is not related to the number of receive antennas $N_{r}$, as opposed to the IDD scheme using the non-decoupled signal model based AB-Log-PDA, which has a computational complexity of $\mathcal{O}\left(4 M N_{t} N_{r}^{2}+2 M N_{t} N_{r}\right)+\mathcal{O}\left(4 N_{t} N_{r}^{2}\right)$ per outer iteration [16], [17]. This implies that the decoupled model based EB-Log-PDA has a lower computational complexity than the non-decoupled model based AB-Log-PDA in the scenario of $N_{r}>N_{t}$, which is particularly important, because $N_{r}>N_{t}$ is a typical configuration for spatial multiplexing 


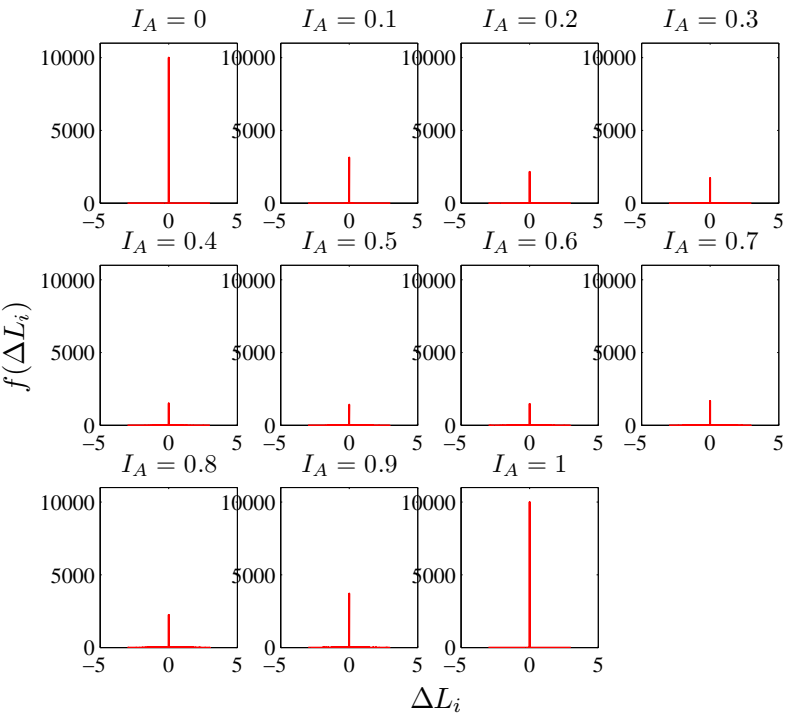

(a) linear scale
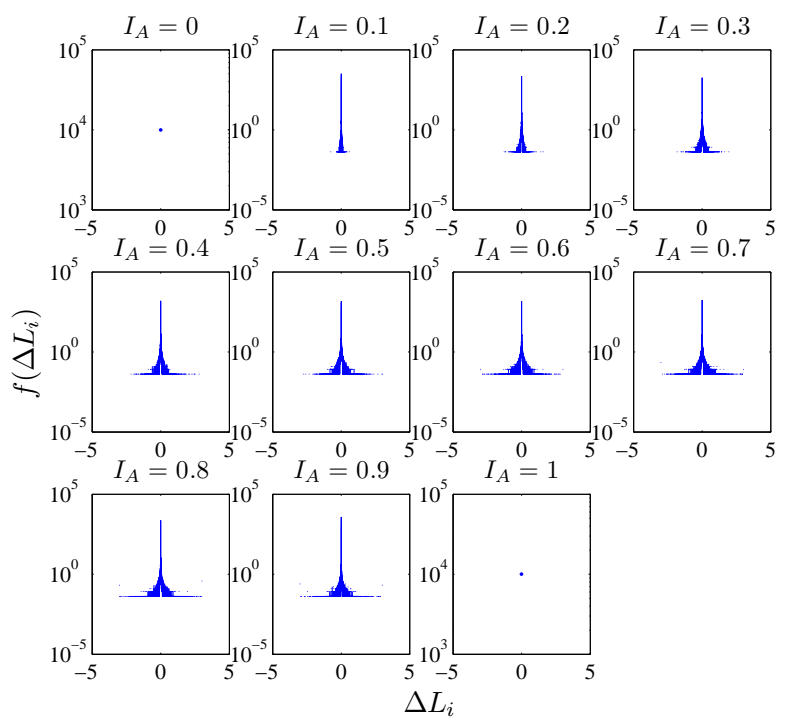

(b) "semilogy" scale

Fig. 10. The probability density functions of $\Delta L_{i}=L_{E}^{E B}(i)-L_{E}^{A B}(i)$, for samples of 240,000 bits, $i t_{i}=0$ inner PDA iteration, and $m=1.0$.

based MIMO systems.

By comparison, the Exact-Log-MAP algorithm has to calculate the Euclidean distance of $\|\mathbf{y}-\mathbf{H s}\|^{2} M^{N_{t}}$ times per bit per outer iteration [13], hence its complexity order is $\mathcal{O}\left(M^{N_{t}} N_{t} \log _{2} M\right)$ per outer iteration. More specifically, the evaluation of $\|\mathbf{y}-\mathbf{H s}\|^{2}$ requires $\mathcal{O}\left(4 N_{r} N_{t}+6 N_{r}\right)$ real operations. Therefore, the Exact-Log-MAP algorithm has a computational complexity of $\mathcal{O}\left[M^{N_{t}} N_{t} \log _{2} M\left(4 N_{r} N_{t}+6 N_{r}\right)\right]$ real operations per outer iteration. There exist other types of reduced-complexity MAP algorithms, such as the look-uptable based Approximate-Log-MAP algorithm and the MaxLog-MAP algorithm [13]. Unfortunately, although they exhibit some advantages in terms of their DSP implementation, in essence both of them are still based on the exhaustive search, hence the computational complexity of calculating the extrinsic LLR for each bit increases exponentially with both the number of transmit antennas $N_{t}$ and the constellation size $M$, because $M^{N_{t}}=2^{M_{b} N_{t}}$ hypotheses have to be evaluated for each bit [13]. Therefore, all the three representative Log-MAP algorithms have a complexity that is significantly higher than that of the EB-Log-PDA, especially when $N_{t}, N_{r}$ and $M$ have large values.

\section{Conclusions}

It was demonstrated in our previous work that the estimated symbol-wise probabilities output by the existing approximate Bayesian theorem based PDA algorithms are not the true APPs, they rather constitute nominal APPs, which are unsuitable for the classic IDD architecture of MIMO systems using general $M$-ary modulations. By contrast, the estimated symbol-wise probabilities at the output of the EB-Log-PDA proposed in this paper exhibit similar characteristics to the true APPs, hence they are readily applicable to the classic IDD architecture of $M$-ary modulation aided MIMO systems. Furthermore, we demonstrated that introducing inner iterations within both the EB-Log-PDA and the AB-Log-PDA, actually degrades the IDD receiver's performance despite significantly increasing the overall computational complexity of the IDD receiver, which implies that the optimal number of inner iterations of both the EB-Log-PDA and the AB-Log-PDA is zero when they are invoked in IDD receivers. Additionally, the relationship between the EB-Log-PDA and the AB-LogPDA was investigated, and it was revealed that the bitwise extrinsic LLRs of the EB-Log-PDA and of the ABLog-PDA are statistically closest to each other, when the mutual information between the bit-sequence output by the interleaver and the corresponding a priori LLRs fed back by the outer FEC decoder approaches its minimum value of 0.0 and maximum value of 1.0 . The difference between the two types of extrinsic LLRs becomes most significant, when the value of the mutual information is in the middle region of the interval $[0,1.0]$. Finally, similar to the case of the AB-Log-PDA based IDD scheme, the IDD scheme based on the proposed EB-Log-PDA using no inner PDA iterations is shown to achieve a comparable performance to that of the optimal MAP detector based IDD receiver, while imposing a significantly lower computational complexity in the scenarios considered.

\section{REFERENCES}

[1] Y. Bar-Shalom and X. R. Li, Estimation and Tracking: Principles, Techniques and Software. Dedham, MA: Artech House, 1993.

[2] Y. Bar-Shalom, F. Daum, and J. Huang, "The probabilistic data association filter," IEEE Control Syst. Mag., vol. 29, no. 6, pp. 82-100, Dec. 2009.

[3] C. Rasmussen and G. D. Hager, "Probabilistic data association methods for tracking complex visual objects," IEEE Trans. Pattern Anal. Mach. Intell., vol. 23, no. 6, pp. 560-576, June 2001.

[4] J. Luo, K. R. Pattipati, P. K. Willett, and F. Hasegawa, "Near optimal multiuser detection in synchronous CDMA using probabilistic data association," IEEE Commun. Lett., vol. 5, no. 9, pp. 361-363, Sept. 2001.

[5] D. Pham, K. R. Pattipati, P. K. Willett, and J. Luo, "A generalized probabilistic data association detector for multiple antenna systems," IEEE Commun. Lett., vol. 8, no. 4, pp. 205-207, Apr. 2004.

[6] S. Liu and Z. Tian, "Near-optimum soft decision equalization for frequency selective MIMO channels," IEEE Trans. Signal Process., vol. 52, no. 3, pp. 721-733, Mar. 2004 . 
[7] — "A Kalman-PDA approach to soft-decision equalization for frequency-selective MIMO channels," IEEE Trans. Signal Process., vol. 53, no. 10, pp. 3819-3830, Oct. 2005.

[8] S. Bavarian and J. K. Cavers, "A new framework for soft decision equalization in frequency selective MIMO channels," IEEE Trans. Commun., vol. 57, no. 2, pp. 415-422, Feb. 2009.

[9] S. Yang, T. Lv, R. G. Maunder, and L. Hanzo, "Unified bit-based probabilistic data association aided MIMO detection for high-order QAM constellations," IEEE Trans. Veh. Technol., vol. 60, no. 3, pp. 981-991, Mar. 2011.

[10] —, "Distributed probabilistic-data-association-based soft reception employing base station cooperation in MIMO-aided multiuser multicell systems," IEEE Trans. Veh. Technol., vol. 60, no. 7, pp. 3532-3538, Sept. 2011.

[11] X. Wang and H. V. Poor, "Iterative (turbo) soft interference cancellation and decoding for coded CDMA," IEEE Trans. Commun., vol. 47, no. 7, pp. 1046-1061, Jul. 1999.

[12] M. Sellathurai and S. Haykin, "Turbo-BLAST for wireless communications: Theory and experiments," IEEE Trans. Signal Process., vol. 50, no. 10, pp. 2538-2546, Oct. 2002.

[13] B. M. Hochwald and S. ten Brink, "Achieving near-capacity on a multiple-antenna channel," IEEE Trans. Commun., vol. 51, no. 3, pp. 389-399, Mar. 2003.

[14] J. Jaldén and B. Ottersten, "On the complexity of sphere decoding in digital communications," IEEE Trans. Signal Process., vol. 53, no. 4, pp. 1474-1484, Apr. 2005

[15] J. Fricke, M. Sandell, J. Mietzner, and P. A. Hoeher, "Impact of the Gaussian approximation on the performance of the probabilistic data association MIMO decoder," EURASIP J. Wireless Commun. Netw., vol. 2005, no. 5, pp. 796-800, 2005.

[16] S. Yang and L. Hanzo, "Iterative detection and decoding using approximate Bayesian theorem based PDA method over MIMO Nakagami$m$ fading channels," in Proc. IEEE Global Commun. Conf. (GLOBECOM'12), Anaheim, CA, USA, Dec. 2012, pp. 3588-3593.

[17] S. Yang, L. Wang, T. Lv, and L. Hanzo, "Approximate Bayesian probabilistic-data-association-aided iterative detection for MIMO systems using arbitrary $M$-ary modulation," IEEE Trans. Veh. Technol., vol. 62, no. 3, pp. 1228-1240, Mar. 2013.

[18] Y. Cai, X. Xu, Y. Cheng, Y. Xu, and Z. Li, "A SISO iterative probabilistic data association detector for MIMO systems," in Proc. 10th International Conf. Commun. Technol. (ICCT'06), Guilin, China, Nov. 2006, pp. 1-4.

[19] J. Proakis, Digital Communications, 4th ed. NY, USA: McGraw-Hill, 2000.

[20] F. D. Neeser and J. L. Massey, "Proper complex random processes with applications to information theory," IEEE Trans. Inf. Theory, vol. 39, no. 4, pp. 1293-1302, July 1993.

[21] T. Adali, P. Schreier, and L. Scharf, "Complex-valued signal processing: The proper way to deal with impropriety," IEEE Trans. Signal Process., vol. 59, no. 11, pp. 5101-5125, Nov. 2011.

[22] P. M. Lee, Bayesian Statistics: An Introduction, 3rd ed. Chichester, UK: Wiley, 2004

[23] S. ten Brink, "Convergence behavior of iteratively decoded parallel concatenated codes," IEEE Trans. Commun., vol. 49, no. 10, pp. 17271737, Oct. 2001.

[24] C. Berrou and A. Glavieux, "Near optimum error correcting coding and decoding: Turbo-codes," IEEE Trans. Commun., vol. 44, no. 10, pp. 1261-1271, Oct. 1996

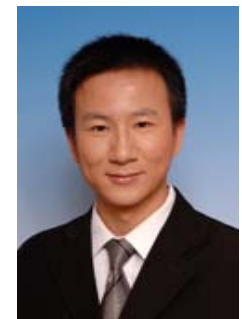

Shaoshi Yang (S'09-M'13) (https://sites.google. com/site/shaoshiyang/) received the B.Eng. Degree in information engineering from Beijing University of Posts and Telecommunications, Beijing, China, in 2006. He is currently working toward the Ph.D. degree in wireless communications with the School of Electronics and Computer Science, University of Southampton, Southampton, U.K., through scholarships from both the University of Southampton and the China Scholarship Council.

From November 2008 to February 2009, he was an Intern Research Fellow with the Communications Technology Laboratory, Intel Labs China, Beijing, where he focused on Channel Quality Indicator Channel design for mobile WiMAX $(802.16 \mathrm{~m})$. His research interests include multiuser detection/multiple-input-mutliple-output (MIMO) detection, multicell joint/distributed processing, cooperative communications, green radio, and interference management. He has published in excess of 20 research papers on IEEE journals and conferences.

Shaoshi is a recipient of the PMC-Sierra Telecommunications Technology Scholarship, and a Junior Member of the Isaac Newton Institute for Mathematical Sciences, Cambridge, UK. He is also a TPC member of both the 23rd Annual IEEE International Symposium on Personal, Indoor and Mobile Radio Communications (IEEE PIMRC 2012), and of the 48th Annual IEEE International Conference on Communications (IEEE ICC 2013).

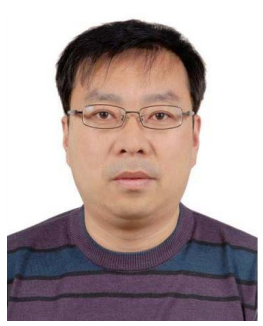

Tiejun Lv (M'08-SM'12) received the M.S. and $\mathrm{Ph} . \mathrm{D}$. degrees in electronic engineering from the University of Electronic Science and Technology of China, Chengdu, China, in 1997 and 2000, respectively. From January 2001 to December 2002, he was a Postdoctoral Fellow with Tsinghua University, Beijing, China. From September 2008 to March 2009, he was a Visiting Professor with the Department of Electrical Engineering, Stanford University, Stanford, CA. He is currently a Professor with the School of Information and Communication Engineering, Beijing University of Posts and Telecommunications. He is the author of more than 100 published technical papers on the physical layer of wireless mobile communications. His current research interests include signal processing, communications theory and networking. Dr. Lv is also a Senior Member of the Chinese Electronics Association. He was the recipient of the "Program for New Century Excellent Talents in University" Award from the Ministry of Education, China, in 2006.

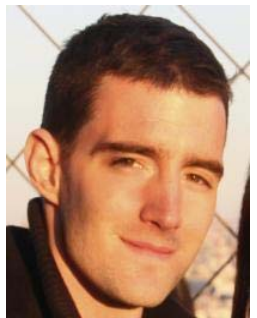

Robert G. Maunder (M'03-SM'13) (http://users. ecs.soton.ac.uk/rm) has studied with Electronics and Computer Science, University of Southampton, UK, since October 2000. He was awarded a first class honors BEng in Electronic Engineering in July 2003, as well as a $\mathrm{PhD}$ in Wireless Communications and a lectureship in December 2007. Rob's research interests include joint source/channel coding, iterative decoding, irregular coding and modulation techniques. He has published a number of IEEE papers in these areas. 


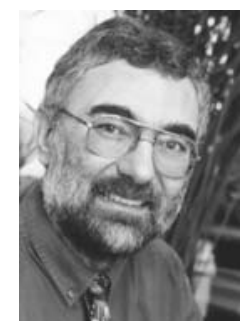

Lajos Hanzo (F'04) received his degree in electronics in 1976 and his doctorate in 1983. In 2004 he was awarded the Doctor of Sciences (DSc) degree by University of Southampton, U.K., and in 2009 he was awarded the honorary doctorate "Doctor Honoris Causa" by the Technical University of $\mathrm{Bu}$ dapest.

During his 36-year career in telecommunications he has held various research and academic posts in Hungary, Germany and the UK. Since 1986 he has been with the School of Electronics and Computer Science, University of Southampton, U.K., where he holds the chair in telecommunications. He has successfully supervised $80 \mathrm{PhD}$ students, coauthored 20 John Wiley/IEEE Press books on mobile radio communications totalling in excess of 10000 pages, published 1300 research entries at IEEE Xplore, acted both as TPC and General Chair of IEEE conferences, presented keynote lectures and has been awarded a number of distinctions. Currently he is directing a 100-strong academic research team, working on a range of research projects in the field of wireless multimedia communications sponsored by industry, the Engineering and Physical Sciences Research Council (EPSRC), U.K., the European IST Programme and the Mobile Virtual Centre of Excellence (VCE), U.K.. He is an enthusiastic supporter of industrial and academic liaison and he offers a range of industrial courses.

Dr. Hanzo is also a Fellow of the Royal Academy of Engineering, and the Institution of Engineering and Technology (IET), as well as the European Association for Signal Processing (EURASIP). He is also a Governor of the IEEE VTS. During 2008-2012 he was the Editor-in-Chief of the IEEE Press and a Chaired Professor at Tsinghua University, Beijing. His research is also funded by the European Research Council's Senior Research Fellow Grant. For further information on research in progress and associated publications please refer to http://www-mobile.ecs.soton.ac.uk 